\title{
Quantization of gravity in the black hole background
}

\author{
Renata Kallosh $\odot^{*}$ and Adel A. Rahman $\oplus^{\dagger}$ \\ Stanford Institute for Theoretical Physics and Department of Physics, Stanford University, \\ Stanford, California 94305, USA
}

(Received 25 June 2021; accepted 9 September 2021; published 5 October 2021)

\begin{abstract}
We perform a covariant (Lagrangian) quantization of perturbative gravity in the background of a Schwarzschild black hole. The key tool is a decomposition of the field into spherical harmonics. We fix Regge-Wheeler gauge for modes with angular momentum quantum number $l \geq 2$, while for low-multipole modes with $l=0$ or 1 -for which Regge-Wheeler gauge is inapplicable-we propose a set of gauge-fixing conditions which are 2D background covariant and perturbatively well defined. We find that the corresponding Faddeev-Popov ghosts are nonpropagating for the $l \geq 2$ modes, but are in general nontrivial for the low-multipole modes with $l=0,1$. However, in Schwarzschild coordinates, all time derivatives acting on the ghosts drop from the action and the low-multipole ghosts have instantaneous propagators. Up to possible subtleties related to quantizing gravity in a space with a horizon, Faddeev's theorem suggests the possibility of an underlying canonical (Hamiltonian) quantization with a manifestly ghost-free Hilbert space.
\end{abstract}

DOI: 10.1103/PhysRevD.104.086008

\section{INTRODUCTION}

We would like to perform a perturbative quantization of gravity in the background of a Schwarzschild black hole, in a setting originally studied by Regge and Wheeler [1], and later studied by Zerilli [2] and Martel and Poisson [3], which was recently discussed in the context of quantization in Refs. [4,5].

As is well known, a key obstacle to a straightforward perturbative quantization of gravity is the presence of a gauge symmetry-diffeomorphism invariance-which leaves the naive Feynman path integral ill defined. A procedure for defining the covariant (Lagrangian) path integral for quantum field theories with gauge symmetries was proposed in Refs. [6,7] and involves breaking the gauge symmetry using a set of gauge-fixing conditions and introducing compensating Faddeev-Popov (FP) ghost fields, whose action is computed according to the rules introduced in Refs. [6,7]. See Appendix A for a review.

In the past, such a quantization of gravity was performed either in a general type of curved background satisfying the Einstein field equations [7-10], with a backgroundcovariant and Lorentz-covariant gauge choice such as background-covariant harmonic gauge, or in a flat Minkowski

\footnotetext{
*kallosh@stanford.edu

†arahman@stanford.edu
}

Published by the American Physical Society under the terms of the Creative Commons Attribution 4.0 International license. Further distribution of this work must maintain attribution to the author(s) and the published article's title, journal citation, and DOI. Funded by SCOAP ${ }^{3}$. background [11-13] with either a Lorentz-covariant gauge choice such as harmonic gauge or a non-Lorentz-covariant gauge choice such as Dirac gauge [14].

In the background-covariant and Lorentz-covariant harmonic gauge and its generalizations, the one- and two-loop computations in gravity were performed in Refs. [8,10,15-18]. In these gauges the FP ghosts are propagating fields which give an important contribution to the Feynman rules. In Refs. $[8,10,17]$ the standard background-covariant and Lorentz-covariant de Donder gauge-fixing condition $F_{\mu}=\nabla_{\nu} h_{\mu}^{\nu}-\frac{1}{2} \nabla_{\mu} h_{\nu}^{\nu}$, linear in the metric perturbation $h_{\mu \nu}$, was used. In Ref. [18] the choice of the gauge-fixing condition involved in addition to the standard de Donder term a set of extra terms nonlinear in $h_{\mu \nu}$ (for example, terms like $h^{\nu \lambda} \nabla_{\lambda} h_{\mu \nu}$ ), with arbitrary, numerical or depending on scalars of the theory, coefficients. The result, in this case of the two-loop UV divergence in gravity, is independent of the seven additional parameters defining the generalized gauge-fixing condition. The FP ghost contributions were computed in these generalized classes of gauges according to standard rules, and the final results were gauge independent, since the FP ghosts were taken into account.

The correct Feynman path integral for computing loop diagrams has been studied in both covariant (Lagrangian) quantization and canonical (Hamiltonian) quantization; see, for example, Refs. [7,11-13,19-21]. We describe the main features of both ways of defining the perturbative Feynman path integral in Appendices A and B, respectively.

Here we briefly discuss the relation between these two types of quantization and their equivalence. In the covariant 
case, the gauge symmetry of the action $S[\phi]=\int \mathcal{L}(\phi)$ with infinitesimal gauge parameters $\xi_{\alpha}(x), \alpha=1, \ldots, m$ has to be broken in order to lift the degeneracy of the kinetic terms and define the propagators. This is accomplished by adding a gauge-fixing condition to the classical theory, for example, in the form $\chi_{\alpha}(\phi)=0$, where the functions $\chi_{\alpha}(\phi)$ depend on the fields $\phi$ of the classical action and possibly their derivatives. The gauge-fixing functions $\chi_{\alpha}$ transform under infinitesimal gauge transformations as

$$
\delta \chi_{\alpha}=Q_{\alpha}^{\beta} \xi_{\beta},
$$

where $Q_{\alpha}{ }^{\beta}$ is in general some differential operator which depends on the fields $\phi$ of the classical action and their derivatives. This then defines the Lagrangian for the FP ghosts corresponding to the given gauge-fixing condition via

$$
\mathcal{L}_{\text {ghost }}[C, \bar{C}]=\bar{C}^{\alpha} Q_{\alpha}{ }^{\beta} C_{\beta},
$$

where $\bar{C}^{\alpha}, C_{\beta}$ are known as FP antighosts and ghosts, respectively. The gauge-fixing condition $\chi_{\alpha}$ is viewed as admissible provided $\operatorname{det}\left\|Q_{\alpha}{ }^{\beta}\right\| \neq 0$ and provided that arbitrary field configurations $\phi$ can always be transformed to configurations satisfying $\chi_{\alpha}(\phi)=0$ via gauge transformations respecting the boundary conditions (see Sec. III C). Covariant quantization has relatively simple rules which guarantee the gauge independence of the physical observables in the theory.

One important feature of the covariant quantization is that the local measure of integration is not strictly defined. We refer to Ref. [20] where this issue was discussed in detail. In particular, in the case that $Q_{\alpha}{ }^{\beta}(\phi)$ is a local function of fields without differential operators acting on $C_{\beta}(x)$, the relevant ghost action simply becomes $\bar{C}^{\alpha}(x) \tilde{C}_{\alpha}(x)$, where $\tilde{C}_{\alpha}(x) \equiv Q_{\alpha}{ }^{\beta}(\phi(x)) C_{\beta}(x)$. The corresponding ghosts are nonpropagating and drop from Feynman rules. The functional determinant resulting from the transformation from $C$ to $\tilde{C}$ may contribute to the local measure of integration some field-dependent divergent term proportional to $\delta^{D}(0)$, where $D$ is the dimension of the spacetime on which $x$ is a coordinate (in our case, we will have $D=2$ since our fields will become effectively twodimensional after decomposition into spherical harmonics). Terms of this nature can be neglected in a regularized theory or can be shown to cancel, as explained in Ref. [20].

The canonical quantization procedure is more fundamental, being based on a canonical Hamiltonian, but it is also more involved. The issue of unitarity of the $S$ matrix can only be addressed in the canonical quantization method. However, according to Refs. [11,13,19-21] the result of covariant quantization is equivalent to the canonical one. In the canonical formulation of theories with gauge symmetries, one encounters first class constraints $\phi^{\alpha}\left(p_{i}, q^{i}\right)=0$, where $i=1, \ldots, n$ runs over the naive configuration space and again $\alpha=1, \ldots, m$. An additional set of conditions $\chi_{\alpha}(p, q)=0$ has to be added to perform the canonical quantization, and it is required that the Poisson brackets of constraints with the additional conditions have a nonvanishing determinant, $\operatorname{det}\left\|\left\{\chi_{\alpha}, \phi^{\beta}\right\}\right\| \neq 0$.

There are two different classes of conditions $\chi_{\alpha}$. One corresponds to the case of "unitary" Hamiltonians in gauge theories, which have manifestly ghost-free underlying Hilbert spaces; the other corresponds to the case of "pseudounitary" Hamiltonians in gauge theories, which have underlying state spaces with negative-norm ghost degrees of freedom which must be quotiented out to yield the physical Hilbert space. In the second case the $S$ matrix is pseudounitary in a space of states with the indefinite metric. More details on this are in Sec. V.

We will show here that in the Regge-Wheeler gauge [1-3] for gravitational perturbations with angular momentum quantum number $l \geq 2$, the corresponding FP ghosts are nonpropagating and decoupled in the covariant quantization method. In the low-multipole $l=1,0$ sector in the two-dimensionally background-covariant gauges we propose here, the FP ghosts are present and in general propagating in covariant quantization. However, in Schwarzschild coordinates in covariant quantization the FP ghosts have "instantaneous" propagators containing only space derivatives, which suggests that our covariant quantization may have a corresponding canonical quantization which belongs to the first class of theories mentioned above, with a unitary Hamiltonian and manifestly ghostfree Hilbert space.

We will provide some evidence that this covariant result is in agreement with one originating from a canonical quantization, as would be expected according to Fadeev's theorem [11]. There may be subtleties in directly applying the logic of Ref. [11] to the situation here, since Schwarzschild coordinates are singular on the event horizon, which was absent in the quantization theorems of Refs. $[11,13,19,20]$. We leave the study and resolution of such subtleties for future work.

\section{GRAVITY IN THE SCHWARZSCHILD BLACK HOLE BACKGROUND}

Consider a 4D asymptotically flat spacetime $\left(\mathcal{M}, \bar{g}_{\mu \nu}\right)$ whose spacetime manifold $\mathcal{M}=\mathcal{M}_{2} \times \mathbb{S}^{2}$ can be endowed with coordinates $\left(x^{a}, \theta^{A}\right), a=1,2$ in which the metric takes the form

$$
\bar{g}_{\mu \nu}=g_{\mu \nu}+h_{\mu \nu}
$$

where $g_{\mu \nu}$ is the metric of the Schwarzschild black hole written in a "spherically symmetric" form

$$
g_{\mu \nu} \mathrm{d} x^{\mu} \mathrm{d} x^{\nu}=g_{a b} \mathrm{~d} x^{a} \mathrm{~d} x^{b}+r^{2}(x) \mathrm{d} \Omega_{2}^{2} .
$$

Here $r(x)$ is defined globally and invariantly by 


$$
4 \pi r(x)^{2} \equiv \operatorname{Area}(\mathrm{SO}(3) \text { orbit of } x \text { in } \mathcal{M})
$$

As noted in Appendix A, we work in the context of 4D asymptotically flat general relativity, for which local diffeomorphisms [i.e., diffeomorphisms obeying certain falloff conditions; see Sec. III C and Eqs. (3.14) and (3.15)] are gauge symmetries which act infinitesimally as

$$
\delta \bar{g}_{\mu \nu}=\bar{\nabla}_{\mu}^{(\bar{g})} \xi_{\nu}+\bar{\nabla}_{\nu}^{(\bar{g})} \xi_{\mu},
$$

where $\bar{\nabla}_{\mu}^{(\bar{g})}$ denotes the (torsion-free) covariant derivative of $\bar{g}_{\mu \nu}$ and where we take the gauge parameter to be the covector $^{1} \xi_{\mu}$. In the context of the background field method within which we will work, the diffeomorphism (2.4) acts on the background $g_{\mu \nu}$ and perturbation $h_{\mu \nu}$ as

$$
\delta g_{\mu \nu}=0, \quad \delta h_{\mu \nu}=\bar{\nabla}_{\mu}^{(\bar{g})} \xi_{\nu}+\bar{\nabla}_{\nu}^{(\bar{g})} \xi_{\mu},
$$

respectively. Note that in this context the diffeomorphisms (2.4) are kept distinct from diffeomorphisms/coordinate transformations of the background $\left(\mathcal{M}, g_{\mu \nu}\right)$.

Our formalism (based on that of Ref. [3]) will be covariant with respect to two-dimensional background diffeomorphisms/coordinate transformations of $\left(\mathcal{M}_{2}, g_{a b}\right)$, i.e., diffeomorphisms/coordinate transformations of the background $\left(\mathcal{M}, g_{\mu \nu}\right)$ which are constant on the two-sphere. Letting $\mathcal{D}_{a}$ and $\epsilon_{a b}$ denote the covariant derivative and volume form of $\left(\mathcal{M}_{2}, g_{a b}\right)$, respectively, it is helpful to define

$$
r_{a}(x)=\mathcal{D}_{a} r(x) \quad \text { and } \quad t^{a}(x)=-\epsilon^{a b} r_{b}(x),
$$

which are orthogonal (but not orthonormal) by construction. Here and below all indices will be raised and lowered with the 2D background metric $g_{a b}$. Here $t^{a}$ is a pseudovector which agrees with the stationary Killing vector of the Schwarzschild background up to its transformation properties under parity $\epsilon_{a b} \rightarrow-\epsilon_{a b}$. Defining

$$
f(r):=g_{a b} r^{a} r^{b}=-g_{a b} t^{a} t^{b}=1-\frac{2 G M}{r},
$$

we have that

$$
g^{a b}=\frac{1}{f(r)}\left(-t^{a} t^{b}+r^{a} r^{b}\right) .
$$

Due to the warp factor $r^{2}(x)$, there are "cross-term" Christoffel symbols for the coordinate systems (2.2) which prevent the simple factorization

$$
\mathrm{d} x^{\mu} \nabla_{\mu} \neq \mathrm{d} x^{a} \mathcal{D}_{a}+\mathrm{d} \theta^{A} D_{A},
$$

\footnotetext{
${ }^{1}$ The corresponding finite gauge transformation is the diffeomorphism generated by $\bar{g}^{\mu \nu} \xi_{\nu}$.
}

where we have let $\nabla_{\mu}$ denote the covariant derivative of $\left(\mathcal{M}, g_{\mu \nu}\right)$ and $D_{A}$ denote the covariant derivative of $\left(\mathbb{S}^{2}, \Omega_{A B}\right)$. These "cross-term" Christoffel symbols are given by

$$
\Gamma_{A B}^{a}=-r g^{a b} r_{b} \Omega_{A B}, \quad \Gamma_{a B}^{\mu}=\frac{1}{r} r_{a} \delta_{B}^{\mu} .
$$

The remaining "cross-term" Christoffel symbols vanish, i.e., $\Gamma_{a b}^{A}=0$.

"Schwarzschild coordinates,"

$$
g_{a b} \mathrm{~d} x^{a} \mathrm{~d} x^{b}=-f(r) \mathrm{d} t^{2}+\frac{\mathrm{d} r^{2}}{f(r)},
$$

which will serve an important role in the latter part of this paper, cover the right static patch (right outer domain of communications) of the Schwarzschild black hole and are adapted to its $\mathbb{Z}_{2} \rtimes \mathbb{R}$ "static $\rtimes$ stationary" isometry. Here the "radial coordinate" $r$ is the function defined by Eq. (2.6) and $t$ is an affine parameter along the timelike orbits of $t^{a}$ which obeys $t^{a} \mathcal{D}_{a} t=1$ in the given patch. It is useful to note that, in these coordinates,

$r_{a} \mathrm{~d} x^{a}=\mathrm{d} r, \quad r^{a} \partial_{a}=f(r) \partial_{r}, \quad t^{a} \partial_{a}=\partial_{t}$.

Note that $t^{a} \partial_{a}=\partial_{t}$ is timelike throughout this patch, asymptoting to a null (pseudo)vector along the event horizon and a unit $t$ translation at infinity. Note that the slices $\left\{\Sigma_{t}\right\}$ of constant $t$ are everywhere spacelike and constitute a family of Cauchy surfaces for this patch.

\section{GRAVITATIONAL PERTURBATIONS IN THE SPHERICAL HARMONIC BASIS}

\section{A. Decomposition into spherical harmonics}

It is helpful to take advantage of the spherical isometries and orientability of the background-and, in particular, of the explicit spherical symmetry of our background gauge choice (2.2) — to expand the metric perturbation $h_{\mu \nu}$ in spherical harmonics of definite parity. In the "spherical" coordinates (2.2), the components of $h_{\mu \nu}$ transform under the action of the background $\mathrm{SO}(3)$ spherical symmetry associated with each two-sphere of fixed $x^{a}$ (and under parity $\epsilon \rightarrow-\epsilon$ ) as three scalars $h_{a b}$, two vectors $h_{a A}$, and one (symmetric) second-order tensor $h_{A B}$. We can thus resolve $h_{\mu \nu}$ into spherical harmonics of definite parity via [1-3]

$$
h_{\mu \nu}=\underbrace{\left(\begin{array}{cc}
p_{a b} & p_{a B}^{(+)} \\
p_{b A}^{(+)} & p_{A B}^{(+)}
\end{array}\right)}_{\text {parity even }}+\underbrace{\left(\begin{array}{cc}
0 & p_{a B}^{(-)} \\
p_{A b}^{(-)} & p_{A B}^{(-)}
\end{array}\right)}_{\text {parity odd }},
$$

with 


$$
\begin{gathered}
p_{a b}=\sum_{\ell=0}^{\infty} \sum_{|m| \leq \ell} h_{a b}^{\ell m} Y^{\ell m}, \\
p_{a A}^{(+)}=\sum_{\ell=1}^{\infty} \sum_{|m| \leq \ell} j_{a}^{\ell m} Y_{A}^{\ell m}, \\
p_{a A}^{(-)}=\sum_{\ell=1}^{\infty} \sum_{|m| \leq \ell} h_{a}^{\ell m} X_{A}^{\ell m}, \\
p_{A B}^{(+)}=r^{2}\left(\sum_{\ell=0}^{\infty} \sum_{|m| \leq \ell} K^{\ell m} \Omega_{A B} Y^{\ell m}+\sum_{\ell=2}^{\infty} \sum_{|m| \leq \ell} G^{\ell m} Y_{A B}^{\ell m}\right) \\
p_{A B}^{(-)}=\sum_{\ell=2}^{\infty} \sum_{|m| \leq \ell} h_{2}^{\ell m} X_{A B}^{\ell m} .
\end{gathered}
$$

Here $Y^{\ell m}\left(\theta^{A}\right)$ are the usual unit-normalized spherical harmonics on $\mathbb{S}^{2}$. The even-parity vector and tensor spherical harmonics are defined by $Y_{A}^{\ell m} \equiv D_{A} Y^{\ell m}$ and $Y_{A B}^{\ell m} \equiv$ $\left[D_{A} D_{B}+\frac{1}{2} \ell(\ell+1) \Omega_{A B}\right] Y^{\ell m}$, respectively. The odd-parity vector and tensor spherical harmonics are defined by $X_{A}^{\ell m} \equiv$ $-\epsilon_{A}^{B} D_{B} Y^{\ell m}$ and $X_{A B}^{\ell m} \equiv-\frac{1}{2}\left[\epsilon_{A}^{C} D_{C} D_{B}+\epsilon_{B}{ }^{C} D_{C} D_{A}\right] Y^{\ell m}$, respectively, where $\epsilon_{A B}$ is the volume form of the round unit sphere and all indices have been raised and lowered with $\Omega_{A B}$.

Note that $p_{a b}$ can be regarded as an $\mathcal{M}_{2}$ tensor and an $\mathbb{S}^{2}$ scalar, $p_{a A}$ as both an $\mathcal{M}_{2}$ vector and an $\mathbb{S}^{2}$ vector, and $p_{A B}$ as an $\mathcal{M}_{2}$ scalar and an $\mathbb{S}^{2}$ tensor. The decomposition (3.1)-(3.4) completely specifies the angular dependence of the perturbation $h_{\mu \nu}$. In particular, the coefficient functions $h_{a b}^{\ell m}, j_{a}^{\ell m}, K^{\ell m}, G^{\ell m}, h_{a}^{\ell m}, h_{2}^{\ell m}$ are "angle independent" (constant on the two-sphere and hence functions only of the two-coordinate $x^{a}$ ) and can be regarded as scalar, vector, and tensor fields on $\mathcal{M}_{2}$.

\section{B. Gauge freedom in spherical harmonics}

Note that, since the different spherical harmonics decouple from one another in the kinetic term of the action, we can make independent gauge choices for each choice of $( \pm) \ell m$. In order to understand how the gauge freedom (2.5)

$$
h_{\mu \nu} \rightarrow h_{\mu \nu}+\bar{\nabla}_{\mu}^{(\bar{g})} \xi_{\nu}+\bar{\nabla}_{\nu}^{(\bar{g})} \xi_{\mu}
$$

acts on a given harmonic, we resolve the gauge parameter $\xi_{\mu}$ into spherical harmonics as

$$
\xi_{\mu}=\underbrace{\left(\Xi_{a}, \Xi_{A}^{(+)}\right)}_{\text {parity even }}+\underbrace{\left(0, \Xi_{A}^{(-)}\right)}_{\text {parity odd }},
$$

with

$$
\begin{array}{r}
\Xi_{a}=\sum_{\ell=0}^{\infty} \sum_{|m| \leq \ell} \xi_{a}^{\ell m} Y^{\ell m}, \\
\Xi_{A}^{(+)}=\sum_{\ell=1}^{\infty} \sum_{|m| \leq \ell} \xi^{(+) \ell m} Y_{A}^{\ell m}, \\
\Xi_{A}^{(-)}=\sum_{\ell=1}^{\infty} \sum_{|m| \leq \ell} \xi^{(-) \ell m} X_{A}^{\ell m} .
\end{array}
$$

$\xi_{a}^{\ell m}, \xi^{(+) \ell m}$, and $\xi^{(-) \ell m}$ are "angle independent" (constant on the two-sphere and hence functions only of the twocoordinate $x^{a}$ ) and can be regarded as scalar and vector fields on $\mathcal{M}_{2}$.

In terms of these variables the gauge transformation (2.5) reads

$\delta p_{a b}=\mathcal{D}_{a} \Xi_{b}+\mathcal{D}_{b} \Xi_{a}-2 \hat{\Gamma}_{a b}^{\mu} \xi_{\mu}$,

$\delta p_{a B}=\mathcal{D}_{a} \Xi_{B}+D_{B} \Xi_{a}-\frac{2}{r} r_{a} \Xi_{b}-2 \hat{\Gamma}_{a B}^{\mu} \xi_{\mu}$,

$\delta p_{A B}=D_{A} \Xi_{B}+D_{B} \Xi_{A}+2 r g^{a b} r_{a} \Xi_{b} \Omega_{A B}-2 \hat{\Gamma}_{A B}^{\mu} \xi_{\mu}$.

The last term involves the linear tensor $\hat{\Gamma}_{\mu \nu}^{\rho}$ which relates the full $\bar{\nabla}_{\mu}^{(\bar{g})}$ and background $\nabla_{\mu}^{(g)}$ covariant derivatives:

$$
\begin{gathered}
\hat{\Gamma}_{\mu \nu}^{\rho} \xi_{\rho} \equiv \bar{\nabla}_{\mu}^{(\bar{g})} \xi_{\nu}-\nabla_{\mu}^{(g)} \xi_{\nu} \\
\hat{\Gamma}_{\mu \nu}^{\rho} \equiv \frac{1}{2} \bar{g}^{\rho \sigma}\left(\nabla_{\mu}^{(g)} h_{\sigma \nu}+\nabla_{\nu}^{(g)} h_{\mu \sigma}-\nabla_{\sigma}^{(g)} h_{\mu \nu}\right) .
\end{gathered}
$$

The key fact that will be useful to us is that such terms do not involve derivatives acting on the gauge parameters $\xi_{\mu}=\left(\Xi_{a}, \Xi^{( \pm)}\right)$, and the contributions of such terms to any FP ghost actions will therefore not involve derivatives acting on ghosts. Since any such terms are at least linear in $h_{\mu \nu}$, they do not contribute to the ghost propagators, but rather provide the couplings for interaction vertices of the form $\bar{C} h^{n} C$, with $n \geq 1$. It is important to stress here that such terms only involve objects which, after decomposing into spherical harmonics, have coefficients which are proper scalars, vectors, or tensors on $\mathcal{M}_{2}$, which can be understood after close inspection of Eq. (3.11).

In general, the terms (3.10) are complicated power series in $h_{\mu \nu}$ which couple different harmonics of the expansions (3.2)-(3.4), (3.7), and (3.8), and outside of the monopole $(l=0)$ sector we do not have explicit closed-form expressions for the sums of these series. The general properties of these terms that we will use are that 1) they do not contribute to ghost propagators and 2) their decomposition into harmonics/2D fields only involves proper representations of the Lorentz group (in particular, the coefficients of all terms in the decomposition and expansion will be proper Lorentz scalars). This latter fact will be used in Appendix C where we study the beyond leading order in $h_{\mu \nu}$ part of the 
$l \geq 1$ ghost actions. These general properties will be sufficient to establish our results. In the monopole $(l=0)$ sector, we have been able to find an explicit closed-form expression [Eq. (4.32)], valid to all orders in perturbation theory which explicitly displays these two properties.

\section{Boundary conditions}

In gravity, the issue of boundary conditions is especially important, since this will affect the asymptotics of the spacetime on top of which our fields propagate, as well as important physical quantities such as the Arnowitt-DeserMisner (ADM) mass and the flux of gravitational radiation measured at infinity. In the context of asymptotically flat gravity that we consider here, this additional consideration places a restriction on the asymptotic falloff of the gauge parameters $\xi_{\mu}$ which relate physically equivalent field configurations of $h_{\mu \nu}$. All other choices of $\xi_{\mu}$ correspond to "large" diffeomorphisms which act nontrivially on the physical space of states. ${ }^{2}$

For 4D general relativity with asymptotically flat boundary conditions ${ }^{3}[13,25,26]$,

$$
h_{\mu \nu} \underset{r \rightarrow \infty}{\sim} O\left(\frac{1}{r}\right), \quad \partial_{\rho} h_{\mu \nu} \underset{r \rightarrow \infty}{\sim} O\left(\frac{1}{r^{2}}\right),
$$

the gauge parameters that relate physically equivalent field configurations of $h_{\mu \nu}$ are those which fall off at least as fast as

$$
\bar{g}^{\mu \nu} \xi_{\nu} \underset{r \rightarrow \infty}{\sim} O\left(\frac{1}{r}\right), \quad \bar{g}^{\mu \nu} \partial_{\rho} \xi_{\nu} \underset{r \rightarrow \infty}{\sim} O\left(\frac{1}{r^{2}}\right),
$$

or, in terms of the decomposition (3.7) and (3.8),

$$
\xi_{a}^{\ell m} \underset{r \rightarrow \infty}{\sim} O\left(\frac{1}{r}\right), \quad \partial_{r} \xi_{a}^{\ell m} \underset{r \rightarrow \infty}{\sim} O\left(\frac{1}{r^{2}}\right)
$$

and

$$
\frac{1}{r^{2}} \xi^{( \pm) \ell m} \underset{r \rightarrow \infty}{\sim} O\left(\frac{1}{r}\right), \quad \frac{1}{r^{2}} \partial_{r} \xi^{( \pm) \ell m} \underset{r \rightarrow \infty}{\sim} O\left(\frac{1}{r^{2}}\right) .
$$

In Eqs. (3.12)-(3.15) we work with the coordinates and radial function (2.6) of the background (2.2), but these statements hold more generally for any asymptotically Minkowskian coordinate system where $r=\sqrt{\|\vec{x}\|^{2}}$ is its spatial radial function. Equation (3.13) can be easily proven

\footnotetext{
${ }^{2}$ An example of a family of such "large" diffeomorphisms which act nontrivially on the physical space of states of asymptotically flat gravity are the BMS supertranslations [22,23]. See, e.g., Ref. [24] for a recent review.

${ }^{3}$ As noted in Ref. [25], these asymptotics are slightly weaker than requiring smoothness at future null infinity $\mathscr{I}^{+}$.
}

using the covariant phase space methods of Refs. [27,28]. The essential point is that an infinitesimal diffeomorphism of an asymptotically flat metric represents a zero mode of the (appropriately restricted) presymplectic form associated with the 4D Einstein-Hilbert action (this is the precise sense in which $\xi_{\mu}$ relates physically equivalent field configurations) if and only if certain surface integrals at spacelike infinity $i_{0}$ vanish. With the asymptotically flat boundary conditions (3.12), this requires the falloff conditions (3.13).

It is important that our gauge-fixing conditions $\chi_{\alpha}$ be chosen such that any field configuration of $h_{\mu \nu}$ can always be put into a form satisfying $\chi_{\alpha}=0$ via a gauge transformation whose generator satisfies Eqs. (3.14) and (3.15). This will ensure that the corresponding gauge slice includes (at least) one representative from each gauge equivalence class of field configurations. We have been careful to check that this is the case for each of the gauge-fixing conditions chosen below.

The falloff conditions (3.14) and (3.15) exclude transformations of the form (3.5) for which the gauge parameters $\xi_{\mu}$ do not vanish at infinity. This clarifies an issue raised in Ref. [3] concerning the gauge fixing of the low multipole $l=0,1$ modes. It was observed in Ref. [3] that their choice of gaugefixing conditions did not seem to fully determine the gauge, since their conditions were preserved by particular families of additional gauge transformations, with particular generators $\xi_{\mu}(v, r)$ presented in ingoing Eddington-Finkelstein (EF) coordinates. However, these generators all violate the conditions (3.14) and (3.15); rather than falling off at large $r$, they are either constant or grow with $r$.

After imposing the falloff conditions (3.14) and (3.15), the gauge-fixing conditions for low multipoles suggested in Ref. [3] in EF coordinates, or the coordinate-independent ones suggested here, fully determine the gauge. ${ }^{4}$ Note that such falloff conditions are also required in quantum field theories with gauge symmetries in order to derive the Ward identities which govern the physical observables.

\section{Regge-Wheeler-Zerilli-Martel-Poisson gauge}

Regge-Wheeler gauge [1] (see also Zerilli [2] and Martel-Poisson [3]) was often used in studies of linearized perturbations of the classical Einstein equations near the Schwarzschild black hole background. We will now review this gauge-fixing condition and its inapplicability for lowmultipole modes with $l=0,1$, in anticipation of its use in covariant quantization in Sec. IV below.

As noted above [see Eqs. (3.1) and (3.4)], after decomposition into spherical harmonics, the gravitational field $h_{\mu \nu}\left(x^{a}, \theta^{A}\right)$ becomes encoded in a set of functions which, as noted above, may be regarded as $2 \mathrm{D}$ fields on $\mathcal{M}_{2}$. In the notation of Ref. [3], these functions/2D fields are

\footnotetext{
${ }^{4}$ They also already fully determine the gauge beyond linear order in $h_{\mu \nu}$.
} 


$$
\begin{array}{rrr}
h_{a b}^{\ell m(+)}, & j_{a}^{\ell m(+)}, & K^{\ell m(+)}, \\
G^{\ell m(+)}, & h_{a}^{\ell m(-)}, & h_{2}^{\ell m(-)} l \geq 2 .
\end{array}
$$

At $l \geq 2$ all functions/2D fields in Eq. (3.16) are available, while for low multipoles only a restricted set of fields is available due to a lack of vector and/or tensor harmonics for those modes. One finds for electric dipoles, magnetic dipoles, and monopoles, respectively, the following fields:

$$
\begin{gathered}
h_{a b}^{1 m(+)}, \quad j_{a}^{1 m(+)}, \quad K^{1 m(+)}, \quad l=1, \quad \text { even, } \\
h_{a}^{1 m(-)}, \quad l=1, \quad \text { odd, } \\
h_{a b}^{00(+)}, \quad K^{00(+)}, \quad l=0 .
\end{gathered}
$$

All functions in Eqs. (3.16)-(3.19) can be regarded as fields on $\mathcal{M}_{2}: h_{a b}$ may be regarded as a tensor on $\mathcal{M}_{2}, j_{a}, h_{a}$ as vectors on $\mathcal{M}_{2}$, and $K, G, h_{2}$ as scalars on $\mathcal{M}_{2}$. The angular dependence of the perturbation $h_{\mu \nu}$ is encoded in the discrete dependence of these fields on the labels $( \pm) \mathrm{lm}$.

As also noted above [see Eqs. (3.7) and (3.8)], after decomposition into spherical harmonics, the gauge parameters $\xi_{\mu}\left(x^{a}, \theta^{A}\right)$ also become encoded in a set of functions which may be regarded as $2 \mathrm{D}$ fields on $\mathcal{M}_{2}$. At $l \geq 2$ for each $l, m$ there are four gauge symmetries. At $l=1(+)$ there are three gauge symmetries for each $m=-1,0,1$, at $l=1(-)$ there is one gauge symmetry for each $m=-1,0$, 1 and at $l=0$ there are two gauge symmetries:

$$
\begin{gathered}
\xi^{l \geq 2} \Rightarrow\left\{\xi_{a}^{\ell m(+)}, \xi^{\ell m(+)}, \xi^{\ell m(-)}\right\}, \\
\xi^{l=1(+)} \Rightarrow\left\{\xi_{a}^{1 m(+)}, \xi^{1 m(+)}\right\}, \\
\xi^{l=1(-)} \Rightarrow\left\{\xi^{1 m(-)}\right\}, \\
\xi^{l=0} \Rightarrow\left\{\xi_{a}^{00(+)}\right\} .
\end{gathered}
$$

All functions in Eqs. (3.20)-(3.23) can similarly be regarded as fields on $\mathcal{M}_{2}: \xi_{a}$ may be regarded as a vector on $\mathcal{M}_{2}$ and $\xi^{( \pm)}$may be regarded as scalars on $\mathcal{M}_{2}$. The angular dependence of the gauge parameter $\xi_{\mu}$ is similarly encoded in the discrete dependence of these fields on the labels $( \pm) \operatorname{lm}$.

The Regge-Wheeler gauge condition for modes with $l \geq 2$ is

$$
j_{a}^{\ell m(+)}=G^{\ell m(+)}=h_{2}^{\ell m(-)}=0 .
$$

It involves one vector and two scalars, leading to a total of four gauge-fixing conditions. One can check that an arbitrary configuration of the fields involved in Eq. (3.24) can always be brought to Regge-Wheeler gauge by means of a gauge transformation whose generator satisfies the falloff conditions (3.14) and (3.15), so these facts together tell us that there is always one and only one field configuration of (the $l \geq 2$ part of) $h_{\mu \nu}$ in each gauge equivalence class which satisfies the Regge-Wheeler gauge condition (3.24). This tells us that Regge-Wheeler gauge is a good gaugefixing condition for covariant quantization. Note that the Regge-Wheeler gauge condition (3.24) is independent of the choice of coordinates $x^{a}$ on $\mathcal{M}_{2}$.

Some of the fields involved in Eq. (3.24) are absent at $l<2$, as one can see in Eqs. (3.17)-(3.19). Therefore, Regge-Wheeler gauge is not a valid gauge choice for lowmultipole modes with $l<2$, and we will present an alternative choice in Sec. IV below.

\section{E. Comments on monopoles $\boldsymbol{l}=\mathbf{0}$ and dipoles $\boldsymbol{l}=\mathbf{1}$}

An important feature of the low-multipole modes established in Refs. $[2,3]$ is the following. First off, the ReggeWheeler gauge is not valid and one has to impose a different set of gauges at $l=0,1$ since for these modes some of the functions in Eq. (3.24) are absent. Examples of such gauge conditions were proposed in Refs. [2,3], where it was also observed that the classical equations of motion for linearized perturbations in this sector have simple localin-time solutions.

This is associated with the feature of the low multipoles $l=0$ and $l=1$ that they do not contain radiative degrees of freedom. The gravitational perturbations near future null infinity were studied in Ref. [3] in the retarded coordinate system $(u, r, \theta, \phi)$, where $u=t-r-2 M \ln (r / 2 M-1)$. It was shown there that the energy carried away by the gravitational radiation near future infinity at $u, r \rightarrow \infty$ is proportional to $l(l-1)$. At the event horizon the radiation was studied in advanced coordinates $(v, r, \theta, \phi)$, where $v=t+r-2 M \ln (r / 2 M-1)$, and again the result is proportional to $l(l-1)$. In both cases the radiation involves quadrupoles, and higher modes, monopoles, and dipoles drop from radiation in agreement with the standard expectation that $l=0$ and $l=1$ perturbations do not contain radiative degrees of freedom.

In the absence of additional sources, all solutions of the Einstein equations for perturbations with $l=0,1$ can be gauged away (according to Refs. [2,3]) by an appropriate choice of coordinate transformation. In the presence of additional sources, like a point particle of mass $m_{0}$ moving towards the black hole, or a particle orbiting a black hole with a fixed angular momentum $a$, solutions for linearized perturbations take a specific form. In the $l=0$ monopole case one finds $h_{t t} \sim \frac{m_{0}}{r}$ [2]. This solution provides a linear correction to the black hole mass proportional to $m_{0}$. In the $l=1$ odd-parity (magnetic dipole) case, the perturbed metric was shown to describe a slightly rotating black hole: one finds (according to Ref. [2]) that the $l=1$ solution for odd-parity perturbations is of the form $\sim \frac{m_{0} a}{r^{2}}$ which represents the linearization of the Kerr metric with 
respect to its angular-momentum parameter, determined here by the angular momentum of the source.

In Ref. [3], where advanced time coordinates were used, the monopole solution was $h_{v v} \sim \frac{2 \delta M}{r}$ and the magnetic dipole solution was $h_{v} \sim \frac{2 \delta J}{r}$. This again confirms that classical solutions for small perturbations lead to small changes in the black hole mass (position of the horizon) and add a small rotation. Finally, for the $l=1$ even-parity (electric dipole) case, solutions for small perturbations are vanishing, even in the presence of sources, and are interpreted as simply encoding a switch to a noninertial coordinate system with respect to the original Schwarzschild spacetime [3].

\section{COVARIANT QUANTIZATION OF GRAVITY IN THE SCHWARZSCHILD BACKGROUND}

\section{A. Gauge-fixed action}

The general form of the BRST-invariant gauge-fixed action $[29,30]$ consists of three terms: the classical action, the gauge-fixing part of the action, and the FP ghost action. In the case of gravity it takes the form

$$
\begin{aligned}
S_{\mathrm{gf}}(g, B, \bar{C}, C ; h)= & S_{\mathrm{cl}}(g+h)+\int B^{\alpha} \chi_{\alpha}(g ; h) \\
& +\int \bar{C}^{\alpha} Q_{\alpha}{ }^{\beta}(g ; h) C_{\beta},
\end{aligned}
$$

where the gauge-fixing conditions $\chi_{\alpha}=0$ result from integrating out the auxiliary fields $B^{\alpha}$. The Feynman path integral acquires the form shown in Eq. (A3).

We now propose the following (two-dimensionally) background-covariant and perturbatively well-defined gauge-fixing conditions, including the Regge-Wheeler case (3.24) as well as a gauge fixing of the low-multipole modes:

$$
\begin{aligned}
j_{a} & =G=h_{2}=0 \quad l \geq 2, \quad \text { even, } \quad \text { odd } \\
K & =j_{a}=0 \quad l=1, \quad \text { even, } \\
r^{a} h_{a} & =0 \quad l=1, \quad \text { odd, } \\
K & =t^{a} r^{b} h_{a b}=0 \quad l=0, \quad \text { even. }
\end{aligned}
$$

All gauge-fixing functions here as well as in Eq. (3.24) are 2D scalars or vectors, with the exception of the case $t^{a} r^{b} h_{a b}=0$ which is a pseudoscalar. This simply means that all auxiliary fields $B^{\alpha}$ for our choice of gauge-fixing functions $\chi_{\alpha}$ are 2D scalars or vectors, with the exception of the case $B t^{a} r^{b} h_{a b}$ where $B$ is a pseudoscalar, so that the total $\int B^{\alpha} \chi_{\alpha}(g ; h)$ contribution to the action is a 2D Lorentz scalar, including the monopole term $B t^{a} r^{b} h_{a b}$. One can check that arbitrary field configurations of $h_{\mu \nu}$ can be made to satisfy Eq. (4.2) by acting with gauge transformations whose generators satisfy Eqs. (3.14) and (3.15). Since Eq. (4.2) also exhausts the gauge freedom (3.5), we see that there is always one and only one field configuration of $h_{\mu \nu}$ in each gauge equivalence class that satisfies the gauge condition Eq. (4.2). This tells us that Eq. (4.2) is a good gauge-fixing condition for covariant quantization.

\section{B. Covariant quantization of the $l \geq 2$ modes: Decoupling of ghosts}

We apply to our theory the DeWitt-Faddeev-Popov procedure $[6,7]$ for the covariant quantization of quantum field theories with gauge symmetries; see Appendix A for a short review. We choose the four Regge-Wheeler gaugefixing conditions used in [1-3] for all modes starting with quadrupoles and above $(l \geq 2)$, is given, for each $(l, m)$, in Eq. (3.24). After writing the gauge symmetry (3.5) in terms of the spherical harmonic decomposition (3.1)-(3.4) for the gravitational field, we will show here that all FP ghosts corresponding to the gauge-fixing conditions (3.24) for even and odd $l \geq 2$ modes decouple.

From Eq. (3.9), we see that the gauge transformation (1.1) of each of the gauge-fixing functions in Eq. (3.24) is

$$
\begin{gathered}
\delta j_{a}^{(+)}=\xi_{a}^{(+)}+\mathcal{D}_{a} \xi^{(+)}-\frac{2}{r} r_{a} \xi^{(+)}+f_{a}^{(+)}[h, \xi], \\
\delta G^{(+)}=\frac{2}{r^{2}} \xi^{(+)}+f_{(G)}^{(+)}[h, \xi] \\
\delta h_{2}^{(-)}=2 \xi^{(-)}+f_{2}^{(-)}[h, \xi] .
\end{gathered}
$$

Here the last terms in these equations $f[h, \xi]$ are functionals of the background metric, the gauge parameters $\xi_{\mu}$, and the metric perturbation $h_{\mu \nu}$ or spacetime derivatives of $h_{\mu \nu}$ which obey $f[0, \xi]=0$ and tensorial linearity in $\xi_{\mu}$ (and hence linearity in $\xi_{a}$ and $\xi^{( \pm)}$, with no dependence on derivatives of $\xi_{a}$ and $\left.\xi^{( \pm)}\right)$. These terms originate from the tensor (3.10) which relates the covariant derivative of the background to the full covariant derivative appearing in Eq. (3.9). As explained above, the contribution to the ghost action due to these terms does not involve derivatives acting on ghosts, and since these terms are at least linear or higher power in $h$, they do not contribute to the ghost propagators, but rather define couplings for the interaction terms $\bar{C} h^{n} C$ with $n \geq 1$.

The total ghost Lagrangian is given by

$$
\begin{aligned}
& \bar{C}^{a}\left(C_{a}^{(+)}+\left(\mathcal{D}_{a}-\frac{2}{r} r_{a}\right) C^{(+)}+f_{a}^{(+)}[h, C]\right) \\
& +\bar{C}^{(+)}\left(\frac{2}{r^{2}} C^{(+)}+f_{(G)}^{(+)}[h, C]\right) \\
& \quad+\bar{C}^{-}\left(2 C^{(-)}+f_{2}^{(-)}[h, C]\right),
\end{aligned}
$$


where we have not specified the details of the interaction terms. The odd-sector ghost action is algebraic and decoupled from the even sector. We can integrate out $\bar{C}^{(-)}$which leads to the constraint $2 C^{(-)}+f_{2}^{(-)}[h, C]=0$, so that the odd ghost action vanishes.

We now integrate over $\bar{C}^{(+)}$to find the constraint

$$
\frac{2}{r^{2}} C^{(+)}+f_{(G)}^{(+)}[h, C]=0 .
$$

The remaining ghost Lagrangian is

$\bar{C}^{a}\left(C_{a}^{(+)}-\frac{r^{2}}{2}\left(\mathcal{D}_{a}-\frac{2}{r} r_{a}\right) f_{(G)}^{(+)}[h, C]+f_{a}^{(+)}[h, C]\right)$,

where we have used Eq. (4.7). To establish the perturbative Feynman rules, we will now look only at the terms quadratic in ghosts and antighosts, without couplings to $h_{\mu \nu}$ (i.e., neglecting terms with $f[h, C]$ ), to find the ghost propagators in the Regge-Wheeler gauge in the background of the Schwarzschild black hole. We find that the terms quadratic in quantum fields are just

$$
\bar{C}^{a(+)} C_{a}^{(+)} .
$$

Thus, the quadratic term for the remaining ghosts is algebraic. We conclude that all ghosts for the ReggeWheeler gauge are nonpropagating and can therefore be neglected for all even and odd $l \geq 2$ modes. We study the nonlinear part of the ghost action in Appendix C where we argue that in Schwarzschild coordinates there are no time derivatives acting on the ghosts at all in the $l \geq 2$ sector.

\section{Covariant quantization of the $l=1$ even (electric dipole) modes}

From Eq. (3.9), we see that the gauge transformation (1.1) of each of the gauge-fixing functions in the $l=1$ even (electric dipole) part of Eq. (4.2) is

$$
\begin{gathered}
\delta j_{a}^{(+)}=\xi_{a}^{(+)}+\mathcal{D}_{a} \xi^{(+)}-\frac{2}{r} r_{a} \xi^{(+)}+f_{a}^{(+)}[h, \xi], \\
\delta K^{(+)}=-\frac{2}{r^{2}} \xi^{(+)}+\frac{2}{r} r^{a} \xi_{a}^{(+)}+f_{(K)}^{(+)}[h, \xi] .
\end{gathered}
$$

The total ghost Lagrangian is therefore given by

$$
\begin{aligned}
& \bar{C}^{a}\left(C_{a}^{(+)}+\left(\mathcal{D}_{a}-\frac{2}{r} r_{a}\right) C^{(+)}+f_{a}^{(+)}[h, C]\right) \\
& +\bar{C}^{(+)}\left(-\frac{2}{r^{2}} C^{(+)}+\frac{2}{r} r^{a} C_{a}^{(+)}+f_{(K)}^{(+)}[h, C]\right) .
\end{aligned}
$$

We integrate over $\bar{C}^{(+)}$to obtain the constraint

$$
C^{(+)}=r r^{a} C_{a}^{(+)}+\frac{r^{2}}{2} f_{(K)}^{(+)}[h, C] .
$$

The remaining ghost Lagrangian is

$$
\begin{aligned}
& \bar{C}^{a}\left(C_{a}^{(+)}+\left(\mathcal{D}_{a}-2 r_{a}\right)\left(r^{a} C_{a}^{(+)}+\frac{r}{2} f_{(K)}^{(+)}[h, C]\right)\right. \\
& \left.\quad+f_{a}^{(+)}[h, C]\right) .
\end{aligned}
$$

The part of this Lagrangian quadratic in ghosts (which defines their propagator) is now

$$
\bar{C}^{a}\left(C_{a}^{(+)}+\left(\mathcal{D}_{a}-2 r_{a}\right) r^{b} C_{b}^{(+)}\right) .
$$

Thus, there is in general a propagating ghost field $C_{a}^{(+)}$.

In Schwarzschild coordinates $(t, r)$, where $r_{a}=(0,1)$, $r^{a}=(0, f)$, Eq. (4.15) becomes

$\bar{C}^{t} C_{t}^{(+)}+\bar{C}^{r} C_{r}^{(+)}+\bar{C}^{t} \mathcal{D}_{t} f C_{r}+\bar{C}^{r}\left(\mathcal{D}_{r}-2\right) f C_{r}^{(+)}$.

This action is linear in $C_{t}^{(+)}$, which we can therefore integrate out to impose the constraint $\bar{C}^{t}=0$. The remaining part of the Lagrangian quadratic in the ghosts is

$$
\bar{C}^{r}\left[\left(\mathcal{D}_{r}-2\right) f+1\right] C_{r}^{(+)} .
$$

The nonlinear part of this Lagrangian is studied in Appendix C where we argue that in Schwarzschild coordinates there are no time derivatives acting on the ghosts in the $l=1$ even sector.

\section{Covariant quantization of the $l=1$ odd (magnetic dipole) modes}

In the $l=1$ odd (magnetic dipole) sector, there is a single gauge symmetry $\xi^{1 m(-)}$ for each $m=-1,0,1$, which we fix using the 2D background-covariant gauge-fixing condition

$$
r^{a} h_{a}^{(-)}=0
$$

From Eq. (3.9), we see that the gauge transformation (1.1) of the gauge-fixing condition is

$$
r^{a} \delta h_{a}^{(-)}=r^{a} \mathcal{D}_{a} \xi^{(-)}+r^{a} f_{a}^{(-)}[h, \xi] .
$$

This variation involves, at the quadratic level defining the ghost kinetic term, a derivative of $\xi^{(-)}$. This means that in general these ghosts are propagating and coupled to gravitational modes. The corresponding ghost action is

$$
\int \bar{C}^{(-)}\left(r^{a}\left(\partial_{a}+V_{a}(h)\right)\right) C^{(-)},
$$


where the coupling $r^{a} V_{a}(h)$ represents vertices where the ghosts for the $l=1$ odd modes interact with $h_{\mu \nu}$.

In Schwarzschild coordinates where $r^{a}=(0, f)$ the ghosts action simplifies to

$$
\bar{C}^{(-)} f\left(\partial_{r}+V_{r}(h)\right) C^{(-)} .
$$

Here the propagator is instantaneous since the kinetic terms have only $r$ derivatives. There are no derivatives acting on this ghost field at higher order in $h_{\mu \nu}$, so in Schwarzschild coordinates there are no time derivatives acting on the ghosts at all in the $l=1$ odd sector.

\section{E. Covariant quantization of the $l=0$ (monopole) modes: Results to all orders}

In the $l=0$ (monopole) sector there is a single 2D vector $\xi_{a}^{00(+)}$, a parameter of a gauge symmetry, which we fix using the $2 \mathrm{D}$ background-covariant gauge-fixing conditions

$$
K=t^{a} r^{b} h_{a b}=0
$$

The first one $(K=0)$ is a 2D scalar, while the second one $\left(t^{a} r^{b} h_{a b}=0\right)$ is a 2D pseudoscalar since $t^{a}=-\epsilon^{a b} r_{b}$ is a pseudovector.

The gauge transformation (1.1) of the gauge-fixing conditions is now given by

$$
\begin{gathered}
t^{a} r^{b} \delta h_{a b}=t^{a} r^{b}\left(\mathcal{D}_{a} \xi_{b}+\mathcal{D}_{b} \xi_{a}+f_{a b}[h, K, \xi]\right), \\
\delta K=\frac{2}{r} r^{a} \xi_{a}+f_{(K)}[h, K, \xi],
\end{gathered}
$$

which leads to a ghost Lagrangian

$$
\begin{aligned}
& \bar{C}^{(K)}\left(\frac{2}{r} r^{a} C_{a}+f_{(K)}[h, C]\right)+\bar{C}^{(h)}\left(t ^ { a } r ^ { b } \left(\mathcal{D}_{a} C_{b}\right.\right. \\
& \left.\left.\quad+\mathcal{D}_{b} C_{a}+f_{a b}[h, C]\right) .\right)
\end{aligned}
$$

We can integrate over $\bar{C}^{(K)}$ which imposes the constraint

$$
\frac{2}{r} r^{a} C_{a}+f_{(K)}[h, C]=0 .
$$

The first term in the ghost action now vanishes. We now note that, since $f_{(K)}[h, C]$ is a Lorentz scalar, it can only depend on $r^{a} C_{a}$ (not the pseudoscalar $t^{a} C_{a}$ ), so that

$$
f_{(K)}[h, C]=\hat{f}_{(K)}[h] r^{a} C_{a}
$$

for some functional $\hat{f}_{(K)}[h]$ of $h_{a b}^{00}$. This means that the constraint (4.27) really reads

$$
\left(\frac{2}{r}+\hat{f}_{(K)}[h]\right) r^{a} C_{a}=0
$$

and so sets $r^{a} C_{a}=0$ up to a local functional determinant. Such changes of variables were discussed in the Introduction below Eq. (1.2) and simply contribute a term proportional to $\delta^{2}(0)$ to the local measure of integration. We neglect these, following the arguments in Ref. [20].

The second term in the Lagrangian is

$$
\bar{C}^{(h)}\left(t^{a} r^{b}\left(\mathcal{D}_{a} C_{b}+\mathcal{D}_{b} C_{a}+f_{a b}[h, C]\right) .\right)
$$

In general, the kinetic term, coming from the part of the action quadratic in ghosts, has both space and time derivatives, and a coupling is present between the ghosts and the 2D monopole sector metric $h_{a b}^{00}$, encoded in the term $\bar{C}^{(h)} t^{a} r^{b} f_{a b}[h, C]$.

However, in Schwarzschild coordinates, we find from Eq. (4.28) that $C_{r}=0$ and Eq. (4.29) reduces to

$$
\bar{C}^{(h)} f(r)\left[\left(\mathcal{D}_{r}-\frac{f^{\prime}}{f}\right) C_{t}+f_{t r}\left[h, C_{t}\right]\right] .
$$

The propagator is again instantaneous since the kinetic terms have only $r$ derivatives, and the nonlinear term $f_{t r}\left[h, C_{t}\right]$ has no derivatives acting on ghosts. Therefore, there are again no time derivatives acting on the ghost fields in Schwarzschild coordinates.

\section{Explicit all orders in $\boldsymbol{h}_{a b}^{00}$ ghost action}

The monopole $l=0$ sector has the advantage that the relevant parts decouple from other modes even at the nonlinear level. Therefore, we are able to find an explicit expression for the ghost action, including all nonlinear terms. With our gauge choices $K=t^{a} r^{b} h_{a b}^{00}=0$ a convenient parametrization for the remaining two components of $h_{a b}$ is

$$
h_{a b}^{00} \equiv \frac{1}{f(r)^{2}} A t_{a} t_{b}+B r_{a} r_{b} .
$$

The Lagrangian for the monopole ghosts is given exactly (to all orders in $h_{\mu \nu}$ ) by

$$
\begin{aligned}
\bar{C}^{(K)} & \left(\frac{2}{r} \frac{1}{1+f(r) B}\right) r^{a} C_{a} \\
+ & \bar{C}^{(h)}\left[\left(t^{a} \partial_{a}-\frac{f(r)\left(t^{b} \partial_{b} B\right)}{1+f(r) B}\right) r^{c} C_{c}\right. \\
& \left.+\left(r^{a} \partial_{a}-\frac{f(r) f^{\prime}(r)-\left(r^{d} \partial_{d} A\right)}{f(r)-A}\right) t^{c} C_{c}\right] .
\end{aligned}
$$

Note that here the antighost field $\bar{C}^{(h)}$ is a $2 \mathrm{D}$ pseudoscalar, the same as the corresponding auxiliary field $B$ in $B t^{a} r^{b} h_{a b}$ 
in Sec. IV A, so the total ghost action is a scalar since $\bar{C}^{(h)}$ multiplies a piece of the Lagrangian which is linear in $t^{a}$.

Now we take into account Eq. (4.28) and we find that the remaining ghost action is given by

$$
\bar{C}^{(h)}\left(r^{a} \partial_{a}-\frac{f(r) f^{\prime}(r)-\left(r^{d} \partial_{d} A\right)}{f(r)-A}\right) t^{c} C_{c} .
$$

In general, this describes propagating ghosts coupled to monopole gravitational perturbations. However, in Schwarzschild coordinates we find only space derivatives acting on ghosts,

$$
\bar{C}^{(h)} f(r)\left(\partial_{r}-\frac{f^{\prime}(r)-\partial_{r} h_{t t}^{00}}{f(r)-h_{t t}^{00}}\right) C_{t} .
$$

Therefore, the propagator is instantaneous, as in the cases above.

\section{FADDEEV'S THEOREM AND A POSSIBLE HAMILTONIAN ORIGIN}

\section{A. Gauges with unitary and pseudounitary Hamiltonians}

In the Hamiltonian formalism in gauge theories where we have first class constraints $\phi^{\alpha}(t, \vec{x})$ and additional conditions $\chi_{\alpha}(t, \vec{x})$, the Poisson bracket

$$
\left\{\chi_{\alpha}(t, \vec{x}), \phi^{\beta}(t, \vec{y})\right\}=M_{\alpha}^{\beta} \delta^{3}(\vec{x}-\vec{y})
$$

defines a differential operator $M_{\alpha}{ }^{\beta}$. As we suggested in the Introduction, there are two different classes of gaugefixing functions $\chi_{\alpha}$ which lead to either a theory with a unitary Hamiltonian, or a theory with the pseudounitary Hamiltonian.

\section{Case of unitary Hamiltonian $\boldsymbol{H}\left(p^{*}, q^{*}\right)$ in gauge theories}

The first class of conditions $\chi_{\alpha}(p, q)$ is usually associated with Lorentz-noncovariant gauges, like Coulomb gauge in Yang-Mills theory or Dirac gauge in gravity. This class includes all instances where the differential operator $M_{\alpha}{ }^{\beta}$ does not involve time derivatives. In such a case, there exists a unitary Hamiltonian in a space with $(n-m)$ physical degrees of freedom $\left(p^{*}, q^{*}\right)$, where $*=1, \ldots,(n-m)$ and which is manifestly ghost free.

In covariant quantization as defined in Ref. [11] one can arrange that

$$
\delta \chi_{\alpha}=-\left\{\chi_{\alpha}, \phi^{\beta}\right\} \xi_{\beta}
$$

at $\chi_{\alpha}=\phi^{\alpha}=0$. Therefore, the differential operator $M_{\alpha}^{\beta}$ defined by the Poisson bracket (5.1) of canonical quantization actually defines the FP ghost action $\bar{C}^{\alpha} Q_{\alpha}{ }^{\beta} C_{\beta}$ in covariant quantization. When the operator $M_{\alpha}{ }^{\beta}$ has only space derivatives, one finds that, though a nontrivial FP ghost action is present in the covariant quantization, it has the particular feature that the ghost propagators are instantaneous.

It is known ${ }^{5}$ in the example of Coulomb gauge in YangMills theory that the ghost loops with instantaneous propagators are canceled by the loops of the instantaneous part of the gluon part of the propagator, to all orders in perturbation theory [32]. It is also known that there are no closed instantaneous loops when the Feynman rules are deduced from the Hamiltonian path integral and the $S$ matrix is computed as the time-ordered product of the unitary Hamiltonian. In this case the equivalence of the Hamiltonian perturbative Feynman rules in QCD $[11,12,31]$ and the Lagrangian DeWitt-Faddeev-Popov rules in QCD is clearly established. In gravity, in the Dirac gauge [14] the ghosts in the covariant quantization also have instantaneous propagators, whereas the underlying Hamiltonian is unitary and the Hilbert space of physical degrees of freedom is ghost free $[11,12]$.

In Appendix B we explain the general relation between the original constrained canonical variables $q$ and $p$ in Eq. (B3) and the independent physical canonical variables $\left(q^{*}, p^{*}\right)$ in Eq. (B9).

\section{Case of pseudounitary Hamiltonian in gauge theories, $\boldsymbol{H}\left(\boldsymbol{q}^{A}, \boldsymbol{p}_{\boldsymbol{A}}, \boldsymbol{\eta}^{a}, \mathcal{P}_{\boldsymbol{a}}\right)$}

The other class involves situations where the condition $\chi_{\alpha}=\chi_{\alpha}(q, p, \lambda, \dot{\lambda})$ depends not only on the naive canonical variables $(q, p)$, but also on Lagrange multipliers $\lambda$ and their time derivative $\dot{\lambda}$, where the Lagrange multipliers originate from the imposition of the constraints in the Hamiltonian form of the action:

$S(q, p, \lambda)=\int \mathrm{d} t\left(p_{i} \dot{q}^{i}-H(q, p)-\lambda_{\alpha} \phi^{\alpha}(q, p)\right)$.

For example, in QCD the field $A_{0}$ is a Lagrange multiplier since the classical action does not depend on $\partial_{0} A^{0}$. In the Lorentz-covariant gauge $\partial_{\mu} A^{\mu}=0$, there is a time derivative of the Lagrange multiplier $\lambda=A^{0}$, since $\partial_{0} A^{0}=\dot{\lambda}$.

The set of canonical coordinates $(q, p)$ is now enlarged by the Lagrange multipliers and their canonical momenta so that the total naive phase space is spanned by $\left(q^{A}, p_{A}\right)$, with $A=1, \ldots, n+m$. This allows for the accommodation of Lorentz-covariant gauges, like Feynman gauge in YangMills theory and de Donder type gauges in gravity. In this case, one must add to the system $2 m$ additional degrees of freedom $\left(\eta^{a}, \mathcal{P}_{a}\right), a=1, \ldots, 2 m$, with opposite statistics,

\footnotetext{
${ }^{5}$ We are grateful to A. Weinstein and M. Shifman for the clarifying discussion of this issue, namely, the absence of ghosts in the canonical Feynman rules in the Coulomb gauge in YangMills theory [11,12,31], and the presence of instantaneous ghosts as well as instantaneous gluons in the covariant Feynman rules.
} 
which correspond to propagating FP ghosts and antighosts. The net number of commuting minus anticommuting canonical variables is $n+m-2 m=n-m$, in agreement with the counting of physical states $\left(q^{*}, p^{*}\right)$ in the unitary gauges described above, where neither Lagrange multipliers nor FP ghosts/antighosts are propagating degrees of freedom. The Hamiltonian in such an extended space $\left(q^{A}, p_{A}, \eta^{a}, \mathcal{P}_{a}\right)$ defines an $S$ matrix which is pseudounitary in a state space with indefinite metric, as explained in Ref. [20].

Examples of gauges with pseudo-unitary Hamiltonian in gravity include harmonic/de Donder gauge, which has propagating (noninstantaneous) FP ghosts in the covariant quantization method. The underlying Hamiltonian in this class of gauges is pseudounitary. The proof of the unitarity of the $S$ matrix in this class of gauges, where ghost degrees of freedom appear even in the canonical/Hamiltonian construction, follows only because of the equivalence of this $S$ matrix to the one in the class of gauges with the ghost-free unitary Hamiltonian.

\section{B. $l \geq 2$}

Our four gauge-fixing functions, for each $(l, m)$ with $l \geq 2$, are $\chi_{\alpha}=\left\{j_{a}, G, h_{2}\right\}$. To leading order in $h_{\mu \nu}$ they transform under the four gauge parameters $\xi_{\alpha}=$ $\left\{\xi_{a}, \xi^{(+)}, \xi^{(-)}\right\}$with the same $l$ and $m$ via

$$
\delta \chi_{\alpha}=-\left\{\chi_{\alpha}, \phi^{\beta}\right\} \xi_{\beta}+O\left(h_{\mu \nu}\right),
$$

where the first term on the right-hand side of Eq. (5.4) is given by the leading-order terms in Eqs. (4.3)-(4.5). The corresponding leading-order ghost action-in particular, the ghost kinetic term-is associated with the determinant of the matrix det $\left\|\left\{\chi_{\alpha}, \phi^{\beta}\right\}\right\| \neq 0$ as explained in Eq. (B5), which is given by

$$
\operatorname{det}\left(\begin{array}{ccc}
\mathbb{1} & \mathcal{D}_{a}-\frac{2}{r} r_{a} & 0 \\
0 & \frac{2}{r^{2}} & 0 \\
0 & 0 & 2
\end{array}\right)=\operatorname{det}\left(\begin{array}{ccc}
\mathbb{1} & 0 & 0 \\
0 & \frac{2}{r^{2}} & 0 \\
0 & 0 & 2
\end{array}\right) .
$$

Such a determinant is algebraic and will contribute to the action as $\delta^{2}(0)$. Therefore, in both covariant and canonical quantization we do not expect propagating ghosts for the modes with $l \geq 2$. One can further show that in Schwarzschild coordinates, time derivatives of ghosts are absent even in the higher-order terms. Thus, the absence of a nontrivial ghost action for the $l \geq 2$ modes provides evidence that a unitary Hamiltonian is available for these modes.

\section{C. $l=1$ even}

Our three gauge-fixing functions, for each $m=-1,0,1$, are $\chi_{\alpha}=\left\{j_{a}, K\right\}$. To leading order they transform under the three gauge parameters $\xi_{\alpha}=\left\{\xi_{a}, \xi^{(+)}\right\}$with the same $m$ via the Poisson bracket $\delta \chi_{\alpha}=-\left\{\chi_{\alpha}, \phi^{\beta}\right\} \xi_{\beta}+O\left(h_{\mu \nu}\right)$ given by the leading-order terms in Eqs. (4.10) and (4.11). The corresponding determinant $\operatorname{det}\left\|\left\{\chi_{\alpha}, \phi^{\beta}\right\}\right\|$ is given by

$$
\begin{aligned}
\operatorname{det}\left(\begin{array}{cc}
1 & \mathcal{D}_{a}-\frac{2}{r} r_{a} \\
\frac{2}{r} r^{a} & -\frac{2}{r^{2}}
\end{array}\right)= & \operatorname{det}\left(-\frac{2}{r^{2}}\right) \\
& -\operatorname{det}\left(\frac{2}{r} r^{a}\left(\mathcal{D}_{a}-\frac{2}{r} r_{a}\right)\right) .
\end{aligned}
$$

In Schwarzschild coordinates, with $r_{a}=(0,1)$ and $r^{a}=(0, f)$, this becomes

$$
\begin{aligned}
\left.\operatorname{det}\left(\begin{array}{cc}
1 & \mathcal{D}_{a}-\frac{2}{r} r_{a} \\
\frac{2}{r} r^{a} & -\frac{2}{r^{2}}
\end{array}\right)\right|_{\text {Schld }}= & \operatorname{det}\left(-\frac{2}{r^{2}}\right) \\
& -\operatorname{det}\left(\frac{2 f(r)}{r}\left(\mathcal{D}_{r}-\frac{2}{r}\right)\right) .
\end{aligned}
$$

Note that this determinant has only space derivatives. Therefore, we can deduce from the canonical quantization in Schwarzschild coordinates that the FP ghosts in a covariant quantization will have an instantaneous propagator. One can further show that in Schwarzschild coordinates, time derivatives of ghosts are absent even in the higher-order terms. Again, the absence of the time derivatives in the ghost action for $l=1$ even modes provides evidence that the unitary Hamiltonian is available for these modes.

\section{D. $l=1$ odd}

Our one gauge-fixing function, for each $m=-1,0,1$, is $\chi_{\alpha}=\left\{r^{a} h_{a}\right\}$ which to leading order transforms under the gauge parameter $\xi_{\alpha}=\left\{\xi^{(-)}\right\}$with the same $m$ via the Poisson bracket $\delta \chi_{\alpha}=-\left\{\chi_{\alpha}, \phi^{\beta}\right\} \xi_{\beta}+O\left(h_{\mu \nu}\right)$ given by the leading-order terms in Eq. (4.19). In Schwarzschild coordinates we see that the corresponding determinant is given by $\operatorname{det}\left\|\left\{\chi_{\alpha}, \phi^{\beta}\right\}\right\|=\operatorname{det}\left(\mathcal{D}_{r}\right)$, which involves a derivative operator in the $r$ direction only. The corresponding FP ghost action shown in Eq. (4.20) is not vanishing but leads to ghost loop diagrams with an instantaneous propagator.

Thus, the ghost action for magnetic dipoles, which has only space derivatives in the action in Schwarzschild coordinates, provides evidence that the unitary Hamiltonian is available for these modes.

\section{E. $l=0$}

In Schwarzschild coordinates we have the two gaugefixing conditions $\chi_{\alpha}=\left\{K, h_{t r}\right\}$ and the two gauge 
parameters $\xi_{r}, \xi_{t}$. The determinant of the corresponding Poisson bracket $\delta \chi_{\alpha}=-\left\{\chi_{\alpha}, \phi^{\beta}\right\} \xi_{\beta}$ is given to all orders in $h_{a b}^{00}$ by

$$
\begin{gathered}
\operatorname{det}\left(\begin{array}{cc}
\frac{2}{r} \frac{f(r)}{1+f(r) h_{r r}} & 0 \\
f(r)\left(\partial_{t}-\frac{f(r) \partial_{t} h_{r r}}{1+f(r) h_{r r}}\right) & f(r)\left(\partial_{r}-\frac{f^{\prime}(r)-\partial_{r} h_{t t}}{f(r)-h_{t t}}\right)
\end{array}\right) \\
=\operatorname{det}\left(\begin{array}{cc}
\frac{2}{r} \frac{f(r)}{1+f(r) h_{r r}} & 0 \\
0 & f(r)\left(\partial_{r}-\frac{f^{\prime}(r)-\partial_{r} h_{t t}}{f(r)-h_{t t}}\right)
\end{array}\right) .
\end{gathered}
$$

Here again we can see that the determinant of the Poisson bracket is in agreement with the expression for the action of the FP ghosts in the covariant method. There are only space derivatives on monopole FP ghosts. Therefore, in the covariant quantization they have instantaneous propagators and it is expected that their loops will cancel with the instantaneous part of the gravitational fields.

Time derivatives in Schwarzschild coordinates explicitly vanish to all orders here. Thus, the ghost action for monopoles, which has only space derivatives in the action, provides evidence that the unitary Hamiltonian is also available in the monopole sector.

\section{DISCUSSION}

A rather surprising feature of the covariant quantization of perturbative gravity in the Schwarzschild black hole background in Regge-Wheeler gauge is that FP ghosts are absent (i.e., decoupled) for all modes with the exception of monopoles and dipoles. These latter modes are known to be related to the ADM mass and angular momentum of the perturbed black hole; for example, on shell and to linear order, they are known to encode the linearized perturbation of the ADM mass and the linearized rotation of the perturbed black hole solution in the presence of sources [1-3].

In the well-known background-covariant and Lorentzcovariant gauges in Refs. $[8,17,18]$ the gauge-fixing functions-e.g., for de Donder gauge $F_{\mu}=\nabla_{\nu}^{(g)} h^{\nu}{ }_{\mu}-$ $\frac{1}{2} \nabla_{\mu}^{(g)} h_{\nu}{ }_{\nu}$-depend on background-covariant derivatives of the gravitational perturbation $h_{\mu \nu}$. The ghosts are propagating due to the tensorial nature of the gravitational perturbations $\delta h_{\mu \nu}=2 \bar{\nabla}_{(\mu}^{(\bar{g})} \xi_{\nu)}$ which involve both space and time derivatives acting on $\xi_{\nu}$. In gravity, Dirac gauge [14] is the only one known to have FP ghosts with instantaneous propagators in a covariant quantization, as shown in Refs. [11-13], and to have a unitary Hamiltonian (in the sense described in Sec. VA above).

Here we have studied covariant quantization of perturbative gravity in a Schwarzschild back hole background, using the Regge-Wheeler [1-3] framework where the quantum fields are expanded in spherical harmonics. In the Schwarzschild black hole background there is a natural split of the space into a warped product $\mathcal{M}=\mathcal{M}_{2} \times \mathbb{S}^{2}$ of two 2D submanifolds. All dependence on the $\mathbb{S}^{2}$ is encoded in the discrete indices of the harmonics, $l, m$ and \pm . For each $(l, m, \pm)$, the quantization is reduced to a quantization of a quasi-twodimensional theory on $\mathcal{M}_{2}$ [with the additional information of a radial function $r(x)$ ]: the gravity perturbations with fixed values of $(l, m, \pm)$ depend only on $x^{a}, a=1,2$, the two coordinates of $\mathcal{M}_{2}$. For low multipoles, for which the Regge-Wheeler gauge condition is not valid, we have proposed a two-dimensionally background-covariant set of gauges in Eq. (4.2). They can can be written out using any choice of coordinates including, for example, Schwarzschild, Eddington-Finkelstein, or KruskalSzekeres coordinates. We found the following.

(1) For all even and all odd modes with $l \geq 2$, the corresponding FP ghosts are not propagating: their kinetic terms do not contain derivatives.

(2) All even and all odd dipole modes with $l=1$, and monopole modes with $l=0$, have nontrivial FP ghosts in covariant quantization, since their kinetic terms involve derivatives.

In the special case of Schwarzschild coordinates our results are as follows.

(1) In Schwarzschild coordinates, the low-multipole ghosts have no time derivatives, only space derivatives. Their propagators are therefore instantaneous, as in Coulomb gauge in QCD [11,12,31] and Dirac gauge in gravity [11-13].

(2) We have provided evidence that with our choice of gauge-fixing functions our covariant quantization rules when viewed in Schwarzschild coordinates are consistent with the existence of an underlying unitary Hamiltonian in a manifestly ghost-free Hilbert space with a positive-definite metric, as suggested by Faddeev's theorem [11].

An open issue which needs to be addressed with regard to a potential canonical quantization of gravity in the black hole background concerns the fact that the existing constructions of the canonical (Hamiltonian) path integral in Refs. [11-13,19,20] were only performed in a flat Minkowski background. Meanwhile, in the black hole background in Schwarzschild coordinates there is an event horizon along which the relevant coordinates break down. The concept of the Hamiltonian and the Hilbert space of states might be more subtle, if well defined at all.

Nevertheless, Faddeev's theorem [11], valid at least in the flat Minkowski background, suggests that a ghost-free unitary Hamiltonian ought to exist for the gauge-fixing conditions studied here in the black hole background in Schwarzschild coordinates. Namely, we have found that in the covariant quantization in Schwarzschild coordinates, 
there are no time derivatives acting on the ghost fields. This suggests that such a Hamiltonian, if explicitly constructed, might belong to the class we described in Sec. VA, the case of the unitary ghost-free Hamiltonian $H\left(p^{*}, q^{*}\right)$ depending on $(n-m)$ degrees of freedom described by $2(n-m)$ canonical variables $\left(p^{*}, q^{*}\right)$.

The reason this is a likely outcome of the canonical quantization is that the case of the pseudounitary Hamiltonian in a Hilbert space of states with an indefinite metric, also described in Sec. VA, $H\left(q^{A}, p_{A}, \eta^{a}, \mathcal{P}_{a}\right)$, would be inconsistent with the absence of time derivatives acting on the ghosts, which we found in this paper. Here, $A=1, \ldots, n+m$ involves commuting fields, and $a=$ $1, \ldots, 2 m$ involves anticommuting fields. The total counting of degrees of freedom is therefore $n+m-2 m=n-m$. But we have just shown that all of our anticommuting fields (FP ghosts and antighosts) have no time derivatives, so they are not expected to contribute to a space of states with an indefinite metric in a process of canonical quantization.

Note that in Eddington-Finkelstein and KruskalSzekeres coordinates the situation is different and has to be studied separately. Although we have performed a covariant quantization which is valid in any of these coordinate systems, the canonical quantization is still to be explored.

To summarize, it would be very interesting to perform a canonical quantization of gravity in the Schwarzschild black hole background in the class of gauges presented here. We leave this for future work.

\section{ACKNOWLEDGMENTS}

We are grateful to A. Barvinsky, R. Bond, V. Chandrasekaran, R. Flauger, A. Linde, R. Mahajan, M. Mirbabayi, E. Poisson, G. Satishchandran, M. Shifman, V. Shyam, E. Silverstein, P. Stamp, D. Stanford, A. Starobinsky, A. Vainshtein, A. Van Proeyen, I. Volovich, and R. Wald for stimulating and helpful discussions. R. K. and A.R. are supported by the Stanford Institute of Theoretical Physics and by the Grant No. PHY-2014215 of National Science Foundation (United States/US). R. K. is additionally supported by the Simons Foundation Origins of the Universe program (Modern Inflationary Cosmology collaboration (United States/US). A. R. is additionally supported by the National Science Foundation GRF Program under Grant No. DGE-1656518 and by a Fletcher Jones Foundation National Science Foundation Graduate Fellowship in the Stanford School of Humanities \& Sciences (United States/US).

\section{APPENDIX A: DEWITT-FADDEEV-POPOV COVARIANT QUANTIZATION OF GRAVITY}

We consider 4D asymptotically flat Einstein gravity in the background of a Schwarzschild black hole $\left(\mathcal{M}, g_{\mu \nu}\right)$. The total classical action depends on $\bar{g}_{\mu \nu}=g_{\mu \nu}+h_{\mu \nu}$, where $h_{\mu \nu}$ is the quantized perturbative gravitational field (graviton field) and the background field is the Schwarzschild black hole metric $g_{\mu \nu}$. The action $S(g+h)$ is invariant under the gauge symmetries

$$
\delta h_{\mu \nu}=\bar{\nabla}_{\mu}^{(g+h)} \xi_{\nu}+\bar{\nabla}_{\nu}^{(g+h)} \xi_{\mu}, \quad \delta g_{\mu \nu}=0,
$$

where $\bar{\nabla}_{\mu}^{(g+h)}$ is the covariant derivative operator of the full metric $g+h$.

Due to the gauge symmetries the naive path integral

$$
\int D h e^{\mathrm{i} S(g+h)}
$$

has to be defined using the DeWitt-Faddeev-Popov procedure [6,7]. This procedure in the simple case suitable for our purpose involves a set of gauge-fixing conditions $\chi_{\alpha}(g ; h)=0$ which constrain the gravitational fields. The path integral becomes

$$
\int D h J_{\chi}(g ; h) \delta\left(\chi_{\alpha}(g ; h)\right) e^{\mathrm{i} S(g+h)}
$$

Here the Jacobian $J_{\chi}(g ; h)$ is defined by the variation of the gauge-fixing function $\chi_{\alpha}(g ; h)$ under the gauge symmetry with the parameters $\xi_{\beta}$,

$$
J_{\chi}(g, h)=\exp \operatorname{Tr} \ln Q_{\alpha}{ }^{\beta}(g, h),
$$

where

$$
\delta \chi_{\alpha}=Q_{\alpha}^{\beta}(g, h) \xi_{\beta}
$$

This Jacobian can also be written with the help of the FP ghosts [6] as follows:

$$
J_{\chi}=\int D \bar{C}^{\alpha} D C_{\beta} e^{\mathrm{i} \int \mathrm{d}^{4} x \bar{C}^{\alpha}(x) Q_{\alpha}^{\beta}(g, h) C_{\beta}(x)}
$$

When $Q_{\alpha}{ }^{\beta}(g ; h)$ is a local function of $(g ; h)$ without differential operators acting on $C_{\beta}(x)$, the relevant ghost action becomes $\bar{C}^{\alpha}(x) \tilde{C}_{\alpha}(x)$, where $\tilde{C}_{\alpha}(x)=Q_{\alpha}{ }^{\beta}(g, h)$ $C_{\beta}(x)$. The corresponding ghosts are nonpropagating and drop from Feynman rules. When $Q_{\alpha}{ }^{\beta}(g ; h)$ involves differential operators with time and space derivatives, the corresponding ghosts are propagating and generically give important contributions to the Feynman diagrams. When $Q_{\alpha}{ }^{\beta}(g ; h)$ involves a differential operator with only space derivatives, the corresponding ghosts are said to have "instantaneous" propagators. 


\section{APPENDIX B: FADDEEV-FRADKIN-VILKOVISKY CANONICAL QUANTIZATION OF GRAVITY}

In this Appendix, we would like to present a brief summary of the most relevant results obtained in Refs. $[11,13,19,20]$ concerning the canonical quantization of gravity. We would like to stress that the standard Faddeev-Fradkin-Vilkovisky quantization of gravity was formulated in the context of a flat Minkowski background, whereas in this paper we perform the quantization in the Schwarzschild black hole background using the covariant quantization method developed by DeWitt [7-10].

It might be useful to clarify the relevant observation made by Weinberg on p. 41 of Ref. [33]. He noticed that in "theories like general relativity there is no way of choosing a coordinate system in which the ghosts decouple. Such theories may be dealt with by the BRST-quantization method described at the end of the previous section, using BRST invariance to prove that the $S$ matrix in a physical ghost-free Hilbert space is unitary."

To clarify this statement we need to explain the precise meaning of the words "the ghosts decouple": one has to make a clear distinction between ghosts decoupling in a covariant BRST quantization procedure and in canonical quantization. For example, one may wonder what exactly the statement above means for, e.g., Dirac gauge [14] in view of the fact that in Refs. [11-13] gravity was canonically and covariantly quantized in the Dirac gauge, with no FP ghosts appearing in the canonical quantization. The clarified statement is: in canonical quantization in Dirac gauge in gravity there is a unitary Hamiltonian and there are no FP ghosts. In covariant quantization in the Dirac gauge, using the BRST method, there are FP ghosts with instantaneous propagators. To explain this we proceed with a review of the results in Refs. [11-13].

The Faddeev-Fradkin-Vilkovisky construction involves defining the canonical (Hamiltonian) Feynman integral for systems with singular Lagrangians, which have the property that the equation

$$
p_{i}=\frac{\partial L(q, \dot{q})}{\partial \dot{q}^{i}}
$$

cannot be solved for $\dot{q}^{i}$ as a function of the $q^{i}$ 's and $p_{i}$ 's, which is a constrained system studied first by Dirac. The Hamiltonian $H\left(q^{i}, p_{i}\right), i=1, \ldots, n$ depends on $2 n$ variables. For such singular Lagrangians with $m$ gauge symmetries, the naive canonical variables $\left(q^{i}, p_{i}\right)$ of the naive phase space $\Gamma$ satisfy a set of first class constraints

$$
\phi^{\alpha}(q, p)=0, \quad \alpha=1, \ldots m .
$$

These are in involution with each other as well as with the Hamiltonian. The constraints define a surface $M$ of dimension $(2 n-m)$ in $\Gamma$.
For such Lagrangians $L(q, \dot{q})$ all equations of motion, including the constraints, can be obtained from the constrained action

$S(q, p, \lambda)=\int \mathrm{d} t\left(p_{i} \dot{q}^{i}-H(q, p)-\lambda_{\alpha} \phi^{\alpha}(q, p)\right)$.

For such systems the Feynman path integral is not well defined unless an additional set of $m$ conditions $\chi_{\alpha}(q, p)$ on the canonical variables is introduced. These conditions, ${ }^{6}$

$$
\chi_{\alpha}(q, p)=0,
$$

define a submanifold $\Gamma^{*}$ in $M$. These functions must satisfy the condition that

$$
\operatorname{det}\left\|\left\{\chi_{\alpha}, \phi^{\beta}\right\}\right\| \neq 0
$$

since only in this case can the surface $\Gamma^{*}$ be defined. Here $\{f, g\}$ are Poisson brackets in $\Gamma$. Here we remind the reader that, as explained near Eq. (5.2), in covariant quantization one can arrange that at $\chi_{\alpha}=\phi^{\alpha}=0$ the variation of the function $\chi_{\alpha}$ is

$$
\delta \chi_{\alpha}=-\left\{\chi_{\alpha}, \phi^{\beta}\right\} \xi_{\beta} .
$$

Therefore, the Poisson bracket

$$
\left\{\chi_{\alpha}(t, \vec{x}), \phi^{\beta}(t, \vec{y})\right\}=M_{\alpha}^{\beta} \delta^{3}(\vec{x}-\vec{y})
$$

of the canonical quantization defines (via $M_{\alpha}{ }^{\beta}$ ) the FP ghost action $\bar{C}^{\alpha} Q_{\alpha}{ }^{\beta} C_{\beta}$ in covariant quantization.

A convenient choice is when $\left\{\chi_{\alpha}, \chi_{\beta}\right\}=0$, in which case we can perform a canonical transformation in $\Gamma$ to obtain the new canonical variables

$$
p_{\alpha}^{\prime}=\chi_{\alpha}(p, q)=0, \quad q^{\prime \alpha}=q^{\prime \alpha}\left(p^{*}, q^{*}\right),
$$

where $q^{\prime \alpha}$ and $p_{\alpha}^{\prime}$ are canonically conjugate, and where the $q^{\prime \alpha}$ are parametrized by the physical canonical variables $q^{*}$, $p^{*}$, which are independent coordinates on $\Gamma^{*}$. Therefore, on $\Gamma^{*}$ we are left with $2(n-m)$ independent canonical variables $\left(p^{*}, q^{*}\right)$. In such a case, the correct Feynman integral can be given in a canonical/Hamiltonian form as

$$
\int \prod_{t} \frac{\mathrm{d} p^{*} \mathrm{~d} q^{*}}{2 \pi} \exp \left\{\mathrm{i}\left[p^{*} \dot{q}^{*}-\mathcal{H}\left(p^{*}, q^{*}\right)\right] \mathrm{d} t\right\}
$$

\footnotetext{
${ }^{6} \mathrm{~A}$ more general choice of conditions is $\chi_{a}(q, p, \lambda, \dot{\lambda})$ where there is a dependence on the Lagrange multiplier and its time derivatives. In these cases the underlying Hamiltonian was also constructed, and depends on $(n+m)$ degrees of freedom. The $S$ matrix in this case is pseudounitary, since the Hilbert space has an indefinite metric [20]. This case includes Lorentz-covariant gauges like a harmonic gauge.
} 
This Hamiltonian defines the unitary $S$ matrix and depends on the $2(n-m)$ physical canonical variables, i.e., on $(n-m)$ physical degrees of freedom. The Hilbert space of physical states has a positive-definite metric and is manifestly ghost free.

The basic result of Faddeev-Fradkin-Vilkovisky in Refs. $[11,13,19,20]$ for the class of constraints in Eq. (B4) is that the path integral in Eq. (B9) based on a unitary Hamiltonian can be given in the form corresponding to a covariant quantization as defined in Refs. [6,7], where the constraint function $\chi_{\alpha}(q, p)$ defines a gaugefixing function $\chi_{\alpha}(\phi)$, depending on the classical fields of the theory. In addition, observables computed using this path integral will be independent of the specific choice of the additional condition/gauge-fixing function.

A simple example of this situation is the Coulomb gauge in QCD. In this case there is a unitary Hamiltonian with only physical degrees of freedom. In the notation of Ref. [11] there is a constraint with the Lagrange multiplier $A^{0}$. The configuration space coordinate is $q^{i}=A^{i}$ with canonical conjugate $p_{k}=E_{k} \equiv F_{0 k}$. The constraint and an additional condition are

$\phi(q, p)=\partial_{k} E_{k}+\left[A_{k}, E_{k}\right], \quad \chi(q, p)=\partial_{i} A_{i}$.

The Poisson bracket $\left\{\chi_{\alpha}, \phi^{\beta}\right\}$ is computed in this case and it gives

$$
\left\{\chi_{\alpha}, \phi^{\beta}\right\}=-\left[\delta^{\alpha \beta} \partial_{i} \partial_{i}+\epsilon^{\alpha \beta \gamma} A_{k}^{\gamma} \partial_{k}\right] \delta^{3}(\vec{x}-\vec{y}) .
$$

It is clear from this example why the determinant of the Poisson bracket at equal times $\left\{\chi_{\alpha}(q, p), \phi^{\beta}(q, p)\right\}$ can only produce space derivatives, and not time derivatives.

The quantization of gravity in Dirac gauge performed in Refs. [11-13] has the same property: there is a unitary Hamiltonian with only physical degrees of freedom. In the covariant Lagrangian quantization there are ghosts, but only with instantaneous propagators.

\section{APPENDIX C: NONLINEAR PART OF THE GHOST ACTION IN SCHWARZSCHILD COORDINATES}

Here we will argue that the higher order in $h_{\mu \nu}$ part of the ghost action corresponding to the gauge-fixing conditions (4.2) does not involve time derivatives acting on ghosts when written in Schwarzschild coordinates.

\section{1. $l \geq 2$ modes}

We expect that the scalar $f_{(G)}^{(+)}[h, C]$ in Eq. (4.4) can depend on the scalars $r^{a} C_{a}$ and $C^{(+)}$(but not on the pseudoscalar $t^{a} C_{a}$ ) so that the constraint (4.7) can be expanded as

$$
\frac{2}{r^{2}} C^{(+)}+\tilde{f}_{(G)}^{(+)}[h] C^{(+)}+\hat{f}_{(G)}^{(+)}[h] r^{b} C_{b}=0,
$$

which allows us to express $C^{(+)}$as a multiple (with an $h_{\mu \nu^{-}}$ dependent coefficient) of $r^{a} C_{a}$. It follows that, after imposing this constraint, $f_{(G)}^{(+)}[h, C]$ depends only on $r^{b} C_{b}$,

$$
f_{(G)}^{(+)}[h, C] \rightarrow-\hat{F}[h] r^{b} C_{b} .
$$

We similarly expect that the vector $f_{a}^{(+)}[h, C]$ appearing in Eq. (4.3) depends on $C_{a}$ and on $r_{a} r^{b} C_{b}$ (since $C^{(+)}$is now a function of $r^{b} C_{b}$ ), i.e., $f_{a}^{(+)}[h, C]=\alpha[h] C_{a}+\beta[h] r_{a} r^{b} C_{b}$, so that the residual ghost action (4.8) reads

$$
\begin{gathered}
\bar{C}^{a}(1+\alpha[h]) C_{a}+\bar{C}^{a}\left(\frac{r^{2}}{2}\left(\mathcal{D}_{a}-\frac{2}{r} r_{a}\right)\right. \\
\left.\times \hat{F}[h] r^{b} C_{b}+\beta[h] r_{a} r^{b} C_{b}\right) .
\end{gathered}
$$

In Schwarzschild coordinates $(t, r)$, where $r_{a}=(0,1)$, $r^{a}=(0, f)$, there is a dependence on $C_{t}$ only in the first term,

$$
\bar{C}^{t}(1+\alpha[h]) C_{t},
$$

so we can integrate over $C_{t}$ to impose the constraint $\bar{C}^{t}=0$, which reduces the ghost action to

$$
\bar{C}^{r} \frac{r^{2}}{2}\left(\mathcal{D}_{r}+\left(\beta[h]-\frac{2}{r}\right)\right) f(r) C_{r} .
$$

We thus find that in Schwarzschild coordinates, the ghost action does not contain any time derivatives acting on the ghost fields even at the higher order in $h_{\mu \nu}$ level. This indicates that the ghost-free unitarizing Hamiltonian may be expected in this sector of the theory.

\section{2. $l=1$ even modes}

The argument is about the same as in $l \geq 2$ case. As argued above, we expect that the higher order in $h_{\mu \nu}$ terms $f[h, C]$ may depend on $\bar{C}^{a}$ contracted with $r_{a} r^{b} C_{b}$ or $C_{a}$. In Schwarzschild coordinates they therefore depend on $\bar{C}^{r} Q[h] C_{r}, \bar{C}^{a} \mathscr{D}_{a} Q[h] C_{r}$, or $\bar{C}^{t} Q[h] C_{t}$, where $Q[h]$ is some functional of $h_{\mu \nu}$ which does not contain differential operators acting on $C_{t}$ or $C_{r}$. We therefore expect that the higher order in $h_{\mu \nu}$ terms will not change the condition $\bar{C}^{t}=0$, which we derived earlier at leading order in $h_{\mu \nu}$. We therefore expect that terms with time derivatives acting on ghosts will not appear at higher orders in $h_{\mu \nu}$, since the only terms with derivatives will be $\bar{C}^{a} \mathcal{D}_{a} C_{r} \rightarrow \bar{C}^{r} \mathcal{D}_{r} C_{r}$. We therefore expect that the higher order in $h_{\mu \nu}$ ghost action for the $l=1$ even modes will be given by an expression analogous to the one in Eq. (C5) with all time derivatives 
acting on the ghosts dropping from this expression in Schwarzschild coordinates.

In the case of $l=1$ odd modes and $l=0$ modes the absence of time derivatives in Schwarzschild coordinates to all orders was already demonstrated in Secs. IV D and IV E, respectively.

\section{APPENDIX D: COMMENTS ON GRAVITATIONAL PROPAGATOR IN THE BLACK HOLE BACKGROUND}

In Ref. [4] an expression for the tensorial part of the gravitational propagator in Regge-Wheeler gauge for the even modes was proposed as

$$
\mathcal{P}^{a b c d}=\frac{1}{4} f_{\ell}\left(k^{2}\right)\left(\eta^{a c} \eta^{b d}+\eta^{a d} \eta^{b c}\right),
$$

where

$f_{\ell}\left(k^{2}\right)=-\frac{4 R_{S}^{2}}{(\lambda+1)}-\frac{2 R_{S}^{4} k^{4}}{(\lambda+1)(\lambda-3)\left(k^{2}+R_{S}^{-2} \lambda\right)}$,

and $\lambda \equiv \ell^{2}+\ell+1$. The relevant part of the effective twodimensional theory was taken in the form

$$
\begin{aligned}
S_{2 d}= & \sum_{l, m} \frac{1}{4} \int \mathrm{d}^{2} x\left(\mathfrak{h}_{l m}^{a b} \Delta_{a b c d}^{-1} \mathfrak{h}_{l m}^{c d}+\mathfrak{h}_{l m}^{a b} \Delta_{L, a b}^{-1} \mathcal{K}_{l m}\right. \\
& \left.+\mathcal{K}_{l m} \Delta_{R, a b}^{-1} \mathfrak{h}_{l m}^{a b}+\mathcal{K}_{l m} \Delta^{-1} \mathcal{K}_{l m}\right)
\end{aligned}
$$

for all $l$. It was important in Eq. (D3) that $\mathcal{K}_{l m} \neq 0$.
Meanwhile, our results show that the action in the cases $l=0,1$ has to be considered separately from $l \geq 2$ cases since in these cases Regge-Wheeler gauge fixing with $\mathcal{K}_{\text {lm }} \neq 0$ is not valid. In particular, as we see from Eq. (4.2) that, for low multipoles $l=0,1$,

$$
\mathcal{K}_{l m}=0, \quad l=0,1
$$

It is therefore not surprising that the propagator in Eqs. (D1)-(D2) has strange properties, noticed in Ref. [4]: the $l=0$ mode causes a change in sign in the second term, and the $l=1$ mode has a pole.

Thus, the derivation of the propagator (D1)-(D2) from the action (D3) is not valid for low multipoles. Instead, for $l=0,1$ one should consider the action (D3) with $\mathcal{K}_{l m}=0$,

$$
S_{2 d}=\sum_{l=0,1 ; m} \frac{1}{4} \int \mathrm{d}^{2} x \mathfrak{h}_{l m}^{a b} \Delta_{a b c d}^{-1} \mathfrak{h}_{l m}^{c d}
$$

Even this action is still a bit dangerous, since the gaugefixing condition $t^{a} r^{b} h_{a b}=0$ still has to be added, to account for the monopole ghosts.

We leave the derivation of the full graviton propagator in the Schwarzschild black hole background for future work.
[1] T. Regge and J. A. Wheeler, Stability of a Schwarzschild singularity, Phys. Rev. 108, 1063 (1957).

[2] F. Zerilli, Gravitational field of a particle falling in a schwarzschild geometry analyzed in tensor harmonics, Phys. Rev. D 2, 2141 (1970).

[3] K. Martel and E. Poisson, Gravitational perturbations of the Schwarzschild spacetime: A Practical covariant and gauge-invariant formalism, Phys. Rev. D 71, 104003 (2005).

[4] N. Gaddam, N. Groenenboom, and G. 't Hooft, Quantum gravity on the black hole horizon, arXiv:2012.02357.

[5] N. Gaddam and N. Groenenboom, Soft graviton exchange and the information paradox, arXiv:2012.02355.

[6] L. Faddeev and V. Popov, Feynman diagrams for the YangMills field, Phys. Lett. 25B, 29 (1967).

[7] B. S. DeWitt, Quantum theory of gravity. 2. The manifestly covariant theory, Phys. Rev. 162, 1195 (1967).

[8] G. 't Hooft and M. Veltman, One loop divergencies in the theory of gravitation, Ann. Inst. H. Poincaré Phys. Theor. A 20, 69 (1974).

[9] R. Kallosh, The renormalization in nonabelian gauge theories, Nucl. Phys. B78, 293 (1974).
[10] M. T. Grisaru, P. van Nieuwenhuizen, and C. Wu, Background field method versus normal field theory in explicit examples: One loop divergences in s matrix and green's functions for yang-mills and gravitational fields, Phys. Rev. D 12, 3203 (1975).

[11] L. D. Faddeev, Feynman integral for singular Lagrangians, Theor. Math. Phys. 1, 1 (1969).

[12] E. S. Fradkin and I. V. Tyutin, S matrix for Yang-Mills and gravitational fields, Phys. Rev. D 2, 2841 (1970).

[13] L. D. Faddeev and V. N. Popov, Covariant quantization of the gravitational field, Sov. Phys. Usp. 16, 777 (1974).

[14] P. A. M. Dirac, Fixation of coordinates in the Hamiltonian theory of gravitation, Phys. Rev. 114, 924 (1959).

[15] R. E. Kallosh, O. V. Tarasov, and I. V. Tyutin, One loop finiteness of quantum gravity off mass shell, Nucl. Phys. B137, 145 (1978).

[16] A. O. Barvinsky and G. A. Vilkovisky, The generalized Schwinger-Dewitt technique in gauge theories and quantum gravity, Phys. Rep. 119, 1 (1985).

[17] M. H. Goroff and A. Sagnotti, The ultraviolet behavior of Einstein gravity, Nucl. Phys. B266, 709 (1986). 
[18] A. van de Ven, Two loop quantum gravity, Nucl. Phys. B378, 309 (1992).

[19] E. S. Fradkin and G. A. Vilkovisky, On renormalization of quantum field theory in curved space-time, Lett. Nuovo Cimento 19, 47 (1977).

[20] E. S. Fradkin and G. A. Vilkovisky, Quantization of relativistic systems with constraints: Equivalence of canonical and covariant formalisms in quantum theory of gravitational field, Lebedev Physical Institute Report No. CERN-TH2332, 1977.

[21] I. A. Batalin and G. A. Vilkovisky, Relativistic S matrix of dynamical systems with boson and fermion constraints, Phys. Lett. B 69, 309 (1977).

[22] H. Bondi, M. G. J. van der Burg, and A. W. K. Metzner, Gravitational waves in general relativity, VII. Waves from Axi-symmetric isolated system, Proc. R. Soc. A 103 (1962).

[23] R. K. Sachs, Gravitational waves in general relativity, VIII. Waves in asymptotically flat space-time, Proc. R. Soc. A 270, 103 (1962).

[24] A. Strominger, Lectures on the Infrared Structure of Gravity and Gauge Theory (Princeton University Press, Princeton, NJ, 2018); See also arXiv:hep-th/1703.05448.
[25] G. Satishchandran and R. M. Wald, The asymptotic behavior of massless fields and the memory effect, Phys. Rev. D 99, 084007 (2019).

[26] R. Arnowitt, S. Deser, and C. W. Misner, Republication of: The dynamics of general relativity, Gen. Relativ. Gravit. 40, 1997 (2008).

[27] J. Lee and R. M. Wald, Local symmetries and constraints, J. Math. Phys. (N.Y.) 31, 725 (1990).

[28] V. Iyer and R. M. Wald, Some properties of noether charge and a proposal for dynamical black hole entropy, Phys. Rev. D 50, 846 (1994).

[29] C. Becchi, A. Rouet, and R. Stora, Renormalization of gauge theories, Ann. Phys. (N.Y.) 98, 287 (1976).

[30] I. V. Tyutin, Gauge invariance in field theory and statistical physics in operator formalism, arXiv:0812.0580.

[31] I. B. Khriplovich, Green's functions in theories with nonabelian gauge group., Sov. J. Nucl. Phys. 10, 235 (1969).

[32] D. Zwanziger, Coulomb - gauge in QCD: Renormalization and confinement, Prog. Theor. Phys. Suppl. 131, 233 (1998).

[33] S. Weinberg, The Quantum Theory of Fields. Vol. 2: Modern Applications (Cambridge University Press, Cambridge, England, 2013). 